\title{
The Cognitive Syndrome of Vascular Dementia: Implications for Clinical Trials
}

\author{
David W. Desmond,*Timo Erkinjuntti, †Mary Sano, †Jeffrey L. Cummings, \\ $\S$ John V. Bowler, $\uparrow$ Florence Pasquier, **Joan T. Moroney, ††Steven H. Ferris, \\ †Yaakov Stern, $†$ Perminder S. Sachdev, and $\S \S$ Vladimir C. Hachinski
}

Department of Neurology, Columbia University, College of Physicians and Surgeons, New York, NY; *Department of Neurology, University of Helsinki, Helsinki, Finland; $\uparrow$ Departments of Neurology and Psychiatry and the Gertrude H. Sergievsky Center, Columbia University, College of Physicians and Surgeons, New York, NY; $†$ Departments of Neurology and Psychiatry \& Biobehavioral Sciences, University of California at Los Angeles School of Medicine, Los Angeles, CA; §Department of Neurology, Royal Free Hospital, London, England; $₫$ Department of Neurology, Memory Clinic, University of Lille, Lille, France; **Department of Neurology and the Gertrude H. Sergievsky Center, Columbia University, College of Physicians and Surgeons, New York, NY; ††Department of Psychiatry, New York University Medical Center, New York, NY; topepartment of Psychiatry, Prince Henry Hospital, University of New South Wales, Little Bay, New South Wales, Australia; §§Department of Clinical Neurological Sciences, University of Western Ontario, London, Ontario, Canada.

Address correspondence to Dr. Desmond, Neurological Institute, 710 West 168th Street, New York, NY 10032. Telephone: (212) 305-1809. Fax: (212) 305-1308. 
Summary: Dementia is common among patients with cerebrovascular disease, particularly in a setting of one or more clinically evident strokes. Prior cohort and case studies have suggested that the cognitive syndrome of vascular dementia is characterized by predominant executive dysfunction, in contrast to the deficits in memory and language function that are typical of patients with Alzheimer disease. The course of cognitive decline may also differ between those dementia subtypes, with many but not all patients with vascular dementia exhibiting a stepwise course of decline due to recurrent stroke and most patients with Alzheimer disease exhibiting a gradually progressive course of decline. The findings of prior studies of the cognitive syndrome of vascular dementia must be interpreted with caution, however, due to possible inaccuracies in the determination of the dementia subtype and the loss of precision that might result from pooling heterogeneous subgroups of patients with vascular dementia, difficulties inherent in identifying a pattern of strengths and weaknesses in patients who are required to have memory impairment and other deficits in order to meet operationalized criteria for dementia, and the use of limited test batteries whose psychometric properties are incompletely understood. Specific questions that should be addressed by future studies are discussed.

Keywords: vascular dementia -- Alzheimer disease -- neuropsychological testing -- clinical trials 


\section{THE COGNITIVE SYNDROME OF VASCULAR DEMENTIA: IMPLICATIONS FOR CLINICAL TRIALS}

Although the importance of the ability to accurately diagnose dementia is readily apparent, the identification of distinctive patterns of cognitive deficits in patients with dementia of different etiologies is also of more than purely intellectual interest (Desmond, 1996). With regard to the specific aims of the conference for which this manuscript was prepared, for example, the recognition of predictable patterns of cognitive deficits could help to guide the selection of efficacy measures for clinical trials in dementia. To that end, this review presents evidence derived from prior cohort and case studies supporting the existence of a cognitive syndrome of vascular dementia, considers some of the methodologic issues that are relevant to studies focusing on that topic, and proposes questions that should be addressed by future studies. Primary distinctions are drawn between vascular dementia and Alzheimer disease because those are the most prevalent dementia subtypes in the general population and present the greatest challenges in differential diagnosis for patient entry into dementia trials. Issues related to patients with coexisting vascular dementia and Alzheimer disease are also addressed. 
Before attempting to determine whether a cognitive syndrome of vascular dementia can be identified, it is worthwhile to consider the frequency with which dementia may be present in patients with cerebrovascular disease. That is, it is informative to determine the "denominator" from which a subgroup of patients with a specific pattern of cognitive deficits might be drawn. Given that patients who have experienced one or more clinically evident ischemic strokes are at greatest risk of vascular dementia and thus may constitute the most appropriate subgroup for inclusion in clinical trials, the findings of the few formal epidemiologic studies that have focused on that subgroup of patients would provide the most useful information. In addition, given that patterns of cognitive deficits are best identified with formal neuropsychological testing, it is important to specifically consider estimates of the frequency of dementia that are derived from studies that administered more rigorous cognitive assessments.

The findings of two prior studies are relevant. In work performed at Columbia-Presbyterian Medical Center in New York, a group of 251 patients age 60 and older who were consecutively hospitalized with acute ischemic stroke was recruited. Three months after stroke, they were examined with neuropsychological tests tapping multiple cognitive domains as well as a measure of functional ability. The diagnosis of dementia was based on criteria modified from the revised third edition of the Diagnostic and Statistical Manual of Mental Disorders (DSM-III-R; American Psychiatric Association, 1987), requiring that subjects exhibit deficits in memory and two or more additional cognitive domains as well as functional impairment. The method for diagnosing vascular dementia was consistent with guidelines later developed by Román et al. (1993). In addition to the presence of cognitive and functional impairment indicative of dementia, it was required that significant cerebrovascular disease be 
evident based on brain imaging, clinical history, and neurologic examination, and that there be a meaningful relationship between those two disorders.

Sixty-six patients, or $26.3 \%$ of the cohort, were demented three months after stroke, compared with $3.2 \%$ of a stroke-free control cohort of comparable age, resulting in an odds ratio of $9.4(95 \%$ confidence interval $=4.2$ to 21.1$)$, adjusted for demographic variables (Tatemichi et al., 1992a). Within the sample of 66 demented patients, $56.1 \%$ had dementia that was directly attributable to stroke, or vascular dementia; $36.4 \%$ had dementia due to the combined effects of stroke and Alzheimer disease, the diagnosis of which was suggested by a pre-stroke history of functional impairment; and 7.5\% had dementia for other reasons, such as alcohol abuse. Recruitment and examination of a second patient cohort is currently being completed at that center, and preliminary analyses have found that the frequency of dementia in the pooled cohorts is virtually identical to that which was recognized in the first cohort. In another large epidemiologic study that was performed in Finland using similar methods, Pohjasvaara et al. (1998) reported that 107 of 337 patients $(31.8 \%)$ met dementia criteria three months after ischemic stroke. Excluding patients with a presumed mixed dementia, 87 of 306 patients $(28.4 \%)$ were felt to have a stroke-related dementia. Although it should be noted that estimates of the frequency of dementia, and thus the determinants of dementia that will be identified, will vary depending on the diagnostic paradigm that is used (Erkinjuntti et al., 1997; Desmond et al., 1998; Pohjasvaara et al., 1998), the results of these two studies are consistent in having found that one-fourth to one-third of elderly patients meet operationalized criteria for dementia three months after stroke, suggesting that dementia after stroke is exceedingly common and warrants 
further exploration to determine whether a characteristic pattern of cognitive deficits can be identified.

\section{Patterns of Cognitive Impairment in Patients with Dementia}

\section{Cohort studies}

Prior studies that have administered comprehensive neuropsychological test batteries to patients with dementia and identified differences in performance between patients with different dementia subtypes have suggested that vascular dementia is characterized by "patchy" deficits (Reichman et al., 1991), or inconsistent patterns of relative strengths and weaknesses between patients. Executive, or frontal lobe, functions tend to be disproportionately impaired (Padovani et al., 1995), however, including planning and sequencing (Villardita, 1993; Kertesz and Clydesdale, 1994; Starkstein et al., 1996), speed of mental processing (Almkvist et al., 1993; Mendez et al., 1997), performance on unstructured tasks (Mendez and Ashla-Mendez, 1991), and attention (Villardita, 1993). Although it has been reported that patients with vascular dementia exhibit greater deficits on measures of verbal fluency than patients with Alzheimer disease (Padovani et al., 1995; Starkstein et al., 1996; Lafosse et al., 1997), most likely due to the frontal 
lobe demands of those tasks, and that the motor aspects of language production may be impaired in patients with vascular dementia (Powell et al., 1988), primary language functions otherwise tend to be preserved. Patients with vascular dementia also exhibit significantly more perseverations than patients with Alzheimer disease, particularly during tasks that assess frontal lobe functions (Lamar et al., 1997).

Patients with Alzheimer disease have been reported to exhibit greater deficits than patients with vascular dementia in functions mediated by posterior cortical structures, such as the temporal and parietal lobes, including memory (Parlato et al., 1988; Loewenstein et al., 1991; Mendez and Ashla-Mendez, 1991; Barr et al., 1992; Gainotti et al., 1992; Del Re et al., 1993; Villardita, 1993; Zimmer et al., 1994; Carlesimo et al., 1995; Erker et al., 1995; Padovani et al., 1995; Batchelder et al., 1997; Hassing and Bäckman, 1997; Lafosse et al., 1997), with a faster rate of information decay (Carlesimo et al., 1993; Carlesimo et al., 1995), a reduced ability to benefit from cues to facilitate retrieval (Del Re et al., 1993; Pasquier et al., 1994), and a higher frequency of intrusion errors (Fuld et al., 1982; Lorscheid and Thompson, 1990; Loewenstein et al., 1991; Barr et al., 1992; Del Re et al., 1993; Lafosse et al., 1997), as well as certain aspects of language function, such as naming (Powell et al., 1988; Barr et al., 1992; Villardita, 1993), which may exacerbate their deficits on verbal memory tasks (Hassing and Bäckman, 1997). It has also been suggested that Alzheimer disease tends to affect lexicon while vascular dementia tends to affect syntax (Hier et al., 1985). When patients with Alzheimer disease exhibit perseverations, they tend to be elicited by tests of semantic knowledge (Lamar et al., 1997). Despite the findings of the above studies, however, it is important to note that a number of prior 
studies have failed to identify significant differences in patterns of cognitive impairment between groups of patients with vascular dementia and Alzheimer disease (e.g., Erkinjuntti et al., 1986).

\section{Case studies}

The pattern of cognitive deficits resulting from stroke may vary dramatically in association with characteristics such as infarct location, number, and size. Thus, it is reasonable to suggest that the findings of cohort studies are more accurate when they are based on patients with a disorder that is more clinically homogeneous, such as Alzheimer disease. In fact, it is possible that the cognitive syndrome of vascular dementia is best illustrated with case reports, particularly with regard to the role of subcortical lesions in dementia.

Although the importance of subcortical gray matter infarctions involving the thalamus and caudate as a basis for cognitive impairment has been demonstrated with case series (Bogousslavsky et al., 1988; Caplan et al., 1990), it has also been reported that certain patients who experience small, deep white matter infarctions may exhibit significant cognitive impairment. Specifically, the syndrome of "strategic infarct dementia" was proposed as a result of the observation of a series of patients with lacunar infarctions involving the inferior capsular genu (Tatemichi et al., 1992b; Tatemichi et al., 1995). Those patients presented with an acute syndrome of confusion and memory loss with striking frontal lobe features; functional brain imaging demonstrated ipsilateral reductions in cortical perfusion, maximal in the frontal and temporal lobes, suggesting disconnection or diaschisis as possible mechanisms for their cognitive impairment. Lacunar infarctions frequently occur in subcortical locations housing or 
neighboring the structures (e.g., thalamus, caudate, globus pallidus) and connecting pathways of frontal-subcortical circuits, accounting at least in part for the reported high frequency of executive dysfunction in patients with vascular dementia and often producing a clinical syndrome similar to that seen in other subcortical diseases (Cummings, 1994). It is also worthy of note that cortical subtypes of strategic infarct dementia can result from insults to the angular gyrus (Benson and Cummings, 1982) and the dorsolateral prefrontal region.

\section{Course of Cognitive Decline in Patients with Dementia}

Although most studies of the cognitive syndrome of vascular dementia have been cross-sectional, patients with vascular dementia may also differ from patients with Alzheimer disease with regard to their course of cognitive decline. Many studies have demonstrated that the typical course of decline in patients with Alzheimer disease is gradual and progressive, but it is probably not truly linear and may vary in rate between and within patients in association with the onset of certain clinical features, such as extrapyramidal signs (Stern et al., 1996a; Stern et al., 1996b). In contrast, little effort has been made to document the presumed stepwise or fluctuating course of decline in vascular dementia. Consistent with the concept of "multi-infarct dementia" (Hachinski et al., 1974) and the results of certain prior prospective studies of risk factors for incident vascular dementia (Loeb et al., 1992; Moroney et al., 1996), that stepwise or fluctuating 
course has been thought to result from multiple recurrent strokes, each of which may cause an acute change in the patient's level of cognitive function followed by a period of stability or partial recovery.

Fischer et al. (1990) performed an informative study of the course of cognitive decline on a sample of patients with either multi-infarct dementia or Alzheimer disease. As expected, they found that $94 \%$ of patients with Alzheimer disease experienced an insidious onset of their dementia syndrome and that $81 \%$ of patients with that dementia subtype exhibited a gradually progressive course of decline, with $81 \%$ of patients with Alzheimer disease exhibiting both of those stereotypic characteristics. Surprisingly, $54 \%$ of patients with multi-infarct dementia experienced the insidious onset of their symptoms and $50 \%$ of patients with that dementia subtype experienced a gradually progressive course of decline. Only $34 \%$ of patients with multi-infarct dementia exhibited both of the stereotypic characteristics of an abrupt onset of dementia followed by a stepwise course of decline.

It has been reported that certain stroke patients exhibit a gradually progressive course of cognitive decline due to clinically "silent" recurrent stroke (Meyer et al., 1995) and exposure to disorders that might result in cerebral hypoxia or ischemia (Moroney et al., 1996). In addition, one-third (Moroney et al., 1997a) to one-half (Yoshitake et al., 1995) of patients with pathologic evidence of vascular dementia lack a history of stroke. Gradually progressive decline in patients with stroke may also be indicative of concomitant Alzheimer disease. Comparative studies of patients with those two dementia subtypes are warranted in order to determine whether features of the course of cognitive decline can reliably facilitate differential diagnosis. 


\section{Methodologic Issues in the Study of the Cognitive Syndrome of Vascular Dementia}

Meaningful conclusions can be drawn from the results of prior studies of the cognitive syndrome of vascular dementia, but certain methodologic issues are worthy of discussion. First, in order for a pattern of cognitive deficits to be identified in patients with vascular dementia, the etiology of the dementia must be correctly determined and groups must be matched for the severity of dementia and other background characteristics, such as age and education. To the extent that patients with mixed dementia, as well as Alzheimer disease or other dementia subtypes (e.g., Lewy body dementia, fronto-temporal dementia), are misdiagnosed with vascular dementia, the inclusion of their cognitive test scores would introduce error into the study findings.

The source from which patients are recruited can have an important impact on the "purity" of the study sample. Vascular dementia patients who are recruited from stroke clinics are likely to be different from vascular dementia patients who are recruited from dementia clinics, where memory loss and the absence of a history of significant cerebrovascular disease are typically required, ensuring an increased frequency of patients whose dementia syndrome is likely to be, to a large extent, determined by Alzheimer disease or other dementia subtypes. Given that the prevalence of both cerebrovascular disease and Alzheimer disease increases with age, however, most patients who are identified due to stroke will fall within an age group that is at increased risk of Alzheimer disease. Thus, some proportion of patients with mixed dementia will also be present in stroke cohorts. 
The differential diagnosis of dementia subtype is a challenging clinical problem, but it is likely that a reliance on operationalized paradigms (Chui et al., 1992; Román et al., 1993) and careful attention to the patient's pre-stroke level of cognitive and functional performance, perhaps through the use of a measure such as the Informant Questionnaire on Cognitive Decline in the Elderly (IQCODE; Jorm and Korten, 1988; Hénon et al., 1997), would help to make that determination. The IQCODE is an informant-based interview that focuses on changes that have occurred during the preceding 10 years in aspects of the patient's daily behavior that place demands on memory and other cognitive functions. Similarly, the Hachinski Ischemic Score (HIS; Hachinski et al., 1975), which was developed to standardize the concepts that commonly guide the differential diagnosis of dementia subtype in clinical practice, has been found to distinguish accurately between patients with pathologically confirmed multi-infarct dementia and dementia due to Alzheimer disease (Moroney et al., 1997a). Operationalized diagnostic paradigms and the HIS do not reliably distinguish patients with those dementia subtypes from patients with mixed dementia (Gold et al., 1997; Moroney et al., 1997a), however, reinforcing the point that histopathologic confirmation is necessary for the most accurate determination of dementia subtype, particularly with regard to degenerative etiologies such as Alzheimer disease. Alternatively, biological markers of Alzheimer disease may help identify patients with combined cerebrovascular disease and Alzheimer-type pathology. Bilateral parietal lobe hypometabolism on positron emission tomography (Jagust, 1998), low levels of amyloid beta protein and elevated levels of tau protein in cerebrospinal fluid (Arai et al., 1995; Motter et al., 1995), and the apolipoprotein E $\varepsilon 4$ genotype (Mayeux et al., 1998) all favor the presence of Alzheimer disease 
and suggest a mixed dementia when recognized in patients with evidence of cerebrovascular disease.

Even when patients with vascular dementia are correctly identified, however, it is possible that patients with different locations and numbers of infarcts could exhibit different patterns of cognitive test scores that might fail to be identified if those subgroups are pooled. For example, a patient with an infarct in the territory of the left posterior cerebral artery involving the temporo-occipital region might present with a syndrome that includes a prominent amnestic state while a patient with an infarct in the territory of the left anterior cerebral artery involving the medial frontal region might present with striking executive dysfunction, but both patients might meet criteria for dementia. Similarly, patients with purely cortical infarcts might exhibit a pattern of cognitive deficits that is different from that of patients with subcortical "strategic infarct dementia." Ultimately, additional studies in which large samples of patients with cerebrovascular disease are administered extensive neuropsychological test batteries may permit the recognition of multiple cognitive syndromes of vascular dementia that vary in association with the specific vascular basis for the dementia syndrome.

Second, a diagnosis of dementia based on certain popular operationalized paradigms (Román et al., 1993; World Health Organization, 1993; American Psychiatric Association, 1994) requires significant impairment in multiple cognitive domains, specifically including memory impairment, biasing a study's results against the recognition of a subtle pattern of relative strengths and weaknesses in cognitive function or a single focal cognitive deficit. For that reason, it might be more informative to attempt to identify patterns of deficits in patients who have not yet met formal criteria for dementia. Similar to the concept of Mild Cognitive 
Impairment (MCI) that was derived from studies of Alzheimer disease (Flicker et al., 1991; Ferris and Kluger, 1996), such patients might be thought to have mild Vascular Cognitive Impairment (VCI; Hachinski, 1994), a far broader term which encompasses the "brain-at-risk" stage through frank dementia. By focusing on those patients, the earliest manifestations of the cognitive syndrome, perhaps most frequently typified by executive dysfunction in patients with cerebrovascular disease, could be identified.

Finally, it is perhaps obvious to emphasize the importance of the assessment tools that are selected for use in the study of the cognitive syndrome of vascular dementia (Desmond et al., 1998). Neuropsychological test batteries must be comprehensive and the psychometric properties of the measures that are chosen should be understood in order to be able to identify valid brain-behavior associations. In short, if all cognitive functions are not assessed or inadequate assessment techniques are used, it will be impossible to fully elicit the pattern of relative strengths and weaknesses that will characterize the cognitive syndrome of vascular dementia.

\section{Questions for Future Research}

Future studies of patients with cerebrovascular disease should address the following questions, which are presented with comments that are not intended to serve as definitive answers: 
(1) How should patients with cerebrovascular disease be screened in order to identify cognitive impairment?

The most popular mental status test remains the Mini-Mental State Examination (MMSE; Folstein et al., 1975), but that scale emphasizes memory and language skills and suffers from a ceiling effect in high functioning patients, an important problem if a trial is intended to focus on patients who are more mildly impaired, such as those with mild VCI. Ideally, a mental status screening test would tap a broader range of cognitive domains with questions that are challenging enough to minimize the possibility of a ceiling effect. The Modified Mini-Mental State (3MS; Teng and Chui, 1987), an expanded MMSE, was developed for that purpose. Another interesting screening test is the Telephone Interview for Cognitive Status (TICS), which was originally developed for use in the assessment of patients with Alzheimer disease who were unwilling or unable to be examined in person (Brandt et al., 1988). In a sample of 36 chronic stroke patients, TICS total score was highly correlated with MMSE total score (0.86), a cutoff score of $<25 / 41$ points was a sensitive (1.00) and specific (0.83) marker for a diagnosis of dementia based on neuropsychological testing and an operationalized paradigm, and the scale did not suffer from a significant ceiling effect (Desmond et al., 1994).

(2) How should cognitive functions be assessed in patients with cerebrovascular disease? Although executive dysfunction is predominant in the cognitive syndrome of vascular dementia, the high frequency of generalized cognitive impairment after stroke suggests that a neuropsychological test battery should take a "modular" form and assess all domains (i.e., memory, orientation, language, and visuospatial function, as well as executive function), with each module containing measures that are relevant to a specific cognitive domain. Scores on 
specific tests within each module could be transformed into z-scores based on the performance of a normative reference group and then averaged to produce a module-specific score.

Module-specific scores could then be averaged to produce a total score for the battery. A module-specific score or the total score on the battery could then be used as a primary or secondary outcome in a vascular dementia trial. The Alzheimer's Disease Assessment Scale (ADAS; Rosen et al., 1984) can be considered to be a model for a battery of this type, but, like the battery later developed by the Consortium to Establish a Registry for Alzheimer's Disease (CERAD; Morris et al., 1989), the tasks originally selected for the ADAS were restricted to those that are specific to the cognitive deficits typically exhibited by patients with Alzheimer disease. Although the ADAS was recently expanded to incorporate cancellation and maze tasks, possibly increasing its utility in the evaluation of executive dysfunction (Mohs et al., 1997), it is essential that researchers ensure that any test that is selected for use in a clinical trial is optimal for the assessment of patients with cerebrovascular disease, and, thus, the efficacy of the therapeutic agent, and not simply a choice made on the basis of convenience.

The selection of specific tests for inclusion within each cognitive domain's module should be dictated by the availability of normative data derived from a compatible reference group free of cerebrovascular disease; the availability of data related to the test's reliability and validity in patients with cerebrovascular disease; the potential for the confounding of test results due to the motor, visual, or auditory demands of the task; and the potential for practice effects in a prospective study. Countless tests have been published (Spreen and Strauss, 1998), but researchers should not hesitate to develop and validate new measures when those that are available are for any reason unsatisfactory. 
A proposal for a comprehensive neuropsychology battery is beyond the scope of this article, but it is worthwhile to consider the possible composition of an executive function module. Regarding subdomains of executive function, the variety of skills that should be assessed include "initiation, planning, hypothesis generation, cognitive flexibility, decision making, regulation, judgment, feedback utilization, and self-perception" (Spreen and Strauss, 1998) as well as attention. The Trail Making Test (Reitan and Wolfson, 1985), which measures mental flexibility and sequencing, or perhaps its orally administered revision (Ricker and Axelrod, 1994; Ricker et al., 1996), is a useful and popular measure, but it would not be sufficient for the assessment of all aspects of executive function. Thus, it should be supplemented with additional measures, such as the Odd Man Out Test (Flowers and Robertson, 1985) or the Short Category Test (Wetzel and Boll, 1987), which measure abstract reasoning and concept formation, and the Digit Span subtest of the Wechsler Adult Intelligence Scale - Revised (Wechsler, 1981) or the Continuous Performance Test, which measure attention (Conners, 1995). To the extent that the assessment of verbal fluency (Benton et al., 1983; Goodglass and Kaplan, 1983 ) is relevant to frontal lobe functioning, it might also be worthwhile to administer a measure of design fluency (e.g., Ruff, 1988), its nonverbal correlate, but it should be noted that the graphomotor demands of such a task would be problematic for some stroke patients. Similarly, the Stroop Color and Word Test (Golden, 1978) is a useful measure of selective attention and cognitive flexibility, but visual acuity and color recognition must be fully intact, and the Wisconsin Card Sorting Test (Heaton et al., 1993) is a popular measure of concept formation, set shifting and set maintenance, and feedback utilization, but it is quite lengthy. The reader should 
consult Spreen and Strauss (1998) for detailed information about these and other measures of executive function.

(3) How should cognitive impairment be staged in patients with cerebrovascular disease?

The Clinical Dementia Rating (CDR; Hughes et al., 1982) is one of the most popular methods for staging the severity of dementia in clinical trials of patients with Alzheimer disease, and it could be useful in trials of patients with cognitive impairment resulting from cerebrovascular disease. Determination of the CDR is based upon an informant's assessment of a patient's cognitive and functional abilities within six domains (i.e., memory, orientation, judgment and problem solving, community affairs, home and hobbies, and personal care) during a structured interview. Domain-specific scores are integrated to produce a global CDR based on an operationalized paradigm, with possible global scores including 0 (no dementia), 0.5 (questionable or very early dementia), 1 (mild dementia), 2 (moderate dementia), and 3 (severe dementia). Given that stroke is a frequent basis for physical disability, however, it is essential that any physical limitations be disregarded when determining whether functional impairment is present. The CDR holds promise as a method for staging cognitive impairment in patients with cerebrovascular disease, but its reliability and validity will need be studied in that population.

(4) Is dementia a valid concept for patients with cerebrovascular disease?

There is value in the ability to identify patients who are most severely affected because their prognosis is likely to be different from that of patients who do not fulfill criteria for dementia (Desmond et al., 1998), but patients with cerebrovascular disease tend to be more heterogeneous than patients with Alzheimer disease with regard to their pattern and relative severities of cognitive deficits. Thus, while the concept of dementia is useful under certain 
circumstances, such as in epidemiologic studies, it may be unduly restrictive under other circumstances. For that reason, it is also worthwhile to conceptualize cognitive impairment along a continuum, like VCI, with an additional separate focus on each of multiple cognitive domains, particularly in the setting of a clinical trial.

(5) Should memory impairment be required for the diagnosis of dementia in patients with cerebrovascular disease?

Although studies have consistently suggested that executive dysfunction is predominant in the cognitive syndrome of vascular dementia, Bowler et al. (1997) found that patients with vascular dementia also exhibit significant memory impairment late in their course of decline. In addition, it has been shown that diagnostic paradigms requiring memory impairment have the greatest predictive validity with regard to the adverse outcomes of recurrent stroke and death among patients with a history of stroke (Desmond et al., 1998). While an operationalized paradigm requiring deficits in memory and other cognitive domains may fail to identify patients who are functionally disabled due to cognitive deficits that do not include memory impairment, it is likely that patients who meet those criteria would be correctly diagnosed with dementia because they would be more advanced in their course of decline. Given that memory impairment is predominant among patients with Alzheimer disease, however, it should be noted that a reliance on such a paradigm would be likely to increase the proportion of patients with mixed dementia who are included in a study sample. As an alternative approach, a diagnostic paradigm could forgo the specification of cognitive deficits required for the diagnosis of dementia and simply consider any number or pattern of cognitive deficits sufficient to cause functional impairment to be supportive of that diagnosis, but that approach would necessitate the 
development of an optimal scale for the functional assessment of patients with cerebrovascular disease, which does not currently exist.

(6) Which patient subgroups should be recruited for inclusion in clinical trials focusing on the treatment of cognitive impairment?

As noted above, given that stroke patients who meet operationalized criteria for dementia are at significantly increased risk of adverse events such as recurrent stroke and death relative to nondemented stroke patients (Tatemichi et al., 1994; Moroney et al., 1997b; Desmond et al., 1998), it is reasonable to suggest that clinical trials should focus on patients who are not yet at highest risk of such a malignant course and who may also be more responsive to treatment, such as those with mild VCI. Alternatively, trials could enroll patients with cerebrovascular disease and all severities of cognitive impairment to determine whether a particular subgroup benefits most from an intervention, or restrict that otherwise unselected sample to patients with solely subcortical disease, which tends to be active and progressive (van Zagten et al., 1996). Given the apparently high frequency with which cerebrovascular disease and Alzheimer disease coexist, clinical trials could have an additional focus on patients with mixed dementia, who might be responsive to Alzheimer-type therapies. For that same reason, trials emphasizing delay to specific endpoints (e.g., decline to a specific cognitive or functional level) may be more appropriate than trials designed to detect improvement in function. 


\section{Acknowledgments}

Presented in part at the Third International Conference on Harmonization of Dementia Drug Guidelines: The Osaka Conference on Vascular Dementia, Osaka, Japan, October 8, 1998. This work was supported in part by grants R01-NS26179, AG10123, and K08-NS02051 from the National Institutes of Health; the Taub Center for Alzheimer's Disease Research at Columbia-Presbyterian Medical Center; an Alzheimer's Disease Research Center of California grant to UCLA; and funding from the Sidell-Kagan Foundation to UCLA. 


\section{References}

Almkvist O, Backman L, Basun H, Wahlund LO. Patterns of neuropsychological performance in Alzheimer's disease and vascular dementia. Cortex 1993; 29: 661-73.

American Psychiatric Association. Diagnostic and statistical manual of mental disorders, 3rd ed., revised. Washington, DC: American Psychiatric Association, 1987.

American Psychiatric Association. Diagnostic and statistical manual of mental disorders, 4th ed. Washington, DC: American Psychiatric Association, 1994.

Arai H, Terajima M, Miura M, et al. Tau in cerebrospinal fluid: a potential diagnostic marker in Alzheimer's disease. Ann Neurol 1995; 38: 649-52.

Barr A, Benedict R, Tune L, Brandt J. Neuropsychological differentiation of Alzheimer's disease from vascular dementia. Int J Geriatr Psychiatry 1992; 7: 621-7.

Batchelder WH, Chosak-Reiter J, Shankle WR, Dick MB. A multinomial modeling analysis of memory deficits in Alzheimer's disease and vascular dementia. J Gerontol B Psychol Sci Soc Sci 1997; 52: 206-15.

Benson DF, Cummings JL. Angular gyrus syndrome simulating Alzheimer's disease. Arch Neurol 1982; 39: 616-20.

Benton A, Hamsher K, Sivan A. Multilingual Aphasia Examination, 3rd ed. Iowa City, IA: AJA Associates, 1983.

Bogousslavsky J, Regli F, Uske A. Thalamic infarcts: clinical syndromes, etiology, and prognosis. Neurology 1988; 38: 837-48. 
Bowler JV, Eliasziw M, Steenhuis R, et al. Comparative evolution of Alzheimer disease, vascular dementia, and mixed dementia. Arch Neurol 1997; 54: 697-703.

Brandt J, Spencer M, Folstein M. The Telephone Interview for Cognitive Status. Neuropsychiatry Neuropsychol Behav Neurol 1988; 1: 111-7.

Caplan LR, Schmahmann JD, Kase CS, et al. Caudate infarcts. Arch Neurol 1990; 47: 133-43.

Carlesimo GA, Fadda L, Bonci A, Caltagirone C. Differential rates of forgetting from long-term memory in Alzheimer's and multi-infarct dementia. Int $J$ Neurosci 1993; 73: 1-11.

Carlesimo GA, Sabbadini M, Fadda L, Caltagirone C. Forgetting from long-term memory in dementia and pure amnesia: role of task, delay of assessment and aetiology of cerebral damage. Cortex 1995; 31: 285-300.

Chui HC, Victoroff JI, Margolin D, Jagust W, Shankle R, Katzman R. Criteria for the diagnosis of ischemic vascular dementia proposed by the State of California Alzheimer's Disease Diagnostic and Treatment Centers. Neurology 1992; 42: 473-80.

Conners C. Conners' Continuous Performance Test. Toronto, Canada: MHS, 1995.

Cummings JL. Vascular subcortical dementias: clinical aspects. Dementia 1994; 5: 177-80.

Del Re ML, Pennese F, Ciurlino P, Abate G. Analysis of verbal memory and learning by means of selective reminding procedure in Alzheimer and multi-infarct dementias. Aging 1993; 5: 185-92.

Desmond DW, Tatemichi T, Hanzawa L. The Telephone Interview for Cognitive Status (TICS): reliability and validity in a stroke sample. Int J Geriatr Psychiatry 1994; 9: 803-7.

Desmond DW. Vascular dementia: a construct in evolution. Cerebrovasc Brain Metab Rev 1996; 8: 296-325. 
Desmond DW, Moroney JT, Bagiella E, Sano M, Stern Y. Dementia as a predictor of adverse outcomes following stroke: an evaluation of diagnostic methods. Stroke 1998; 29: 69-74.

Erker GJ, Searight HR, Peterson P. Patterns of neuropsychological functioning among patients with multi-infarct and Alzheimer's dementia: a comparative analysis. Int Psychogeriatr 1995; 7: 393-406.

Erkinjuntti T, Laaksonen R, Sulkava R, Syrjalainen R, Palo J. Neuropsychological differentiation between normal aging, Alzheimer's disease and vascular dementia. Acta Neurol Scand 1986; 74: 393-403.

Erkinjuntti T, Ostbye T, Steenhuis R, Hachinski V. The effect of different diagnostic criteria on the prevalence of dementia. N Engl J Med 1997; 337: 1667-74.

Ferris S, Kluger A. Commentary on Age-Associated Memory Impairment, Age-Related Cognitive Decline and Mild Cognitive Impairment. Aging Neuropsychol Cogn 1996; $3: 148-153$.

Fischer P, Gatterer G, Marterer A, Simanyi M, Danielczyk W. Course characteristics in the differentiation of dementia of the Alzheimer type and multi-infarct dementia. Acta Psychiatr Scand 1990; 81: 551-3.

Flicker C, Ferris SH, Reisberg B. Mild cognitive impairment in the elderly: predictors of dementia. Neurology 1991; 41: 1006-9.

Flowers KA, Robertson C. The effect of Parkinson's disease on the ability to maintain a mental set. J Neurol Neurosurg Psychiatry 1985; 48: 517-29.

Folstein MF, Folstein SE, McHugh PR. "Mini-mental state": a practical method for grading the cognitive state of patients for the clinician. J Psychiatr Res 1975; 12: 189-98. 
Fuld PA, Katzman R, Davies P, Terry RD. Intrusions as a sign of Alzheimer dementia: chemical and pathological verification. Ann Neurol 1982; 11: 155-9.

Gainotti G, Parlato V, Monteleone D, Carlomagno S. Neuropsychological markers of dementia on visual-spatial tasks: a comparison between Alzheimer's type and vascular forms of dementia. J Clin Exp Neuropsychol 1992; 14: 239-52.

Gold G, Giannakopoulos P, Montes-Paixao C, et al. Sensitivity and specificity of newly proposed clinical criteria for possible vascular dementia. Neurology 1997; 49: 690-4.

Golden J. Stroop Color and Word Test. Chicago, IL: Stoelting Company, 1978.

Goodglass H, Kaplan E. The assessment of aphasia and related disorders, 2nd ed. Philadelphia, PA: Lea \& Febiger, 1983.

Hachinski V. Vascular dementia: a radical redefinition. Dementia 1994; 5: 130-2.

Hachinski VC, Iliff LD, Zilhka E, et al. Cerebral blood flow in dementia. Arch Neurol $1975 ; 32: 632-7$

Hachinski VC, Lassen NA, Marshall J. Multi-infarct dementia: a cause of mental deterioration in the elderly. Lancet 1974; $2: 207-10$.

Hassing L, Bäckman L. Episodic memory functioning in population-based samples of very old adults with Alzheimer's disease and vascular dementia. Dement Geriatr Cogn Disord 1997; 8: 376-83.

Heaton R, Chelune G, Talley J, Kay G, Curtis G. Wisconsin Card Sorting Test (WCST) manual, revised and expanded. Odessa, FL: Psychological Assessment Resources, 1993.

Hénon H, Pasquier F, Durieu I, et al. Preexisting dementia in stroke patients: baseline frequency, associated factors, and outcome. Stroke 1997; 28: 2429-36. 
Hier DB, Hagenlocker K, Shindler AG. Language disintegration in dementia: effects of etiology and severity. Brain Lang 1985; 25: 117-33.

Hughes CP, Berg L, Danziger WL, Coben LA, Martin RL. A new clinical scale for the staging of dementia. Br J Psychiatry 1982; 140: 566-72.

Jagust W. Neuroimaging of dementing disorders. In: Markesbery W, editor. Neuropathology of dementing disorders. New York: Arnold, 1998: 25-55.

Jorm AF, Korten AE. Assessment of cognitive decline in the elderly by informant interview. Br J Psychiatry 1988; 152: 209-13.

Kertesz A, Clydesdale S. Neuropsychological deficits in vascular dementia vs Alzheimer's disease: frontal lobe deficits prominent in vascular dementia. Arch Neurol 1994; 51: 1226-31.

Lafosse JM, Reed BR, Mungas D, Sterling SB, Wahbeh H, Jagust WJ. Fluency and memory differences between ischemic vascular dementia and Alzheimer's disease. Neuropsychology 1997; 11: 514-522.

Lamar M, Podell K, Carew TG, et al. Perseverative behavior in Alzheimer's disease and subcortical ischemic vascular dementia. Neuropsychology 1997; 11: 523-534.

Loeb C, Gandolfo C, Croce R, Conti M. Dementia associated with lacunar infarction. Stroke 1992; 23: 1225-9.

Loewenstein DA, D'Elia L, Guterman A, et al. The occurrence of different intrusive errors in patients with Alzheimer's disease, multiple cerebral infarctions, and major depression. Brain Cogn 1991; 16: 104-17. 
Lorscheid T, Thompson SL. Intrusion errors in patients with degenerative or vascular dementia: the cholinergic connection? Dementia 1990; 1: 202-7.

Mayeux R, Saunders AM, Shea S, et al. Utility of the apolipoprotein E genotype in the diagnosis of Alzheimer's disease: Alzheimer's Disease Centers Consortium on Apolipoprotein E and Alzheimer's Disease. N Engl J Med 1998; 338: 506-11.

Mendez MF, Ashla-Mendez M. Differences between multi-infarct dementia and Alzheimer's disease on unstructured neuropsychological tasks. J Clin Exp Neuropsychol 1991; 13: 923-32.

Mendez MF, Cherrier MM, Perryman KM. Differences between Alzheimer's disease and vascular dementia on information processing measures. Brain Cogn 1997; 34: 301-10.

Meyer JS, Muramatsu K, Mortel KF, Obara K, Shirai T. Prospective CT confirms differences between vascular and Alzheimer's dementia. Stroke 1995; 26: 735-42.

Mohs RC, Knopman D, Petersen RC, et al. Development of cognitive instruments for use in clinical trials of antidementia drugs: additions to the Alzheimer's Disease Assessment Scale that broaden its scope. The Alzheimer's Disease Cooperative Study. Alzheimer Dis Assoc Disord 1997; 11: S13-21.

Moroney JT, Bagiella E, Desmond DW, et al. Meta-analysis of the Hachinski Ischemic Score in pathologically verified dementias. Neurology 1997a; 49: 1096-105.

Moroney JT, Bagiella E, Desmond DW, Paik MC, Stern Y, Tatemichi TK. Risk factors for incident dementia after stroke: role of hypoxic and ischemic disorders.

Stroke 1996; 27: 1283-9. 
Moroney JT, Bagiella E, Tatemichi TK, Paik MC, Stern Y, Desmond DW. Dementia after stroke increases the risk of long-term stroke recurrence. Neurology 1997b; 48: 1317-25.

Morris JC, Heyman A, Mohs RC, et al. The Consortium to Establish a Registry for Alzheimer's Disease (CERAD). Part I. Clinical and neuropsychological assessment of Alzheimer's disease. Neurology 1989; 39: 1159-65.

Motter R, Vigo-Pelfrey C, Kholodenko D, et al. Reduction of beta-amyloid peptide 42 in the cerebrospinal fluid of patients with Alzheimer's disease. Ann Neurol 1995; 38: 643-8.

Padovani A, Di Piero V, Bragoni M, Iacoboni M, Gualdi GF, Lenzi GL. Patterns of neuropsychological impairment in mild dementia: a comparison between Alzheimer's disease and multi-infarct dementia. Acta Neurol Scand 1995; 92: 433-42.

Parlato V, Carlomagno S, Merla F, Bonavita V. Patterns of verbal memory impairment in dementia: Alzheimer disease versus multinfarctual dementia. Acta Neurol 1988; 10: 343-51.

Pasquier F, Jacob B, Lefebvre L, Grymonprez L, Debachy B, Petit H. How to evaluate cognitive dysfunction in patients with vascular dementia? In: Leys D and Scheltens P, editors. Vascular dementia. Dordrecht, The Netherlands: ICG Publications, 1994: 47-53.

Pohjasvaara T, Erkinjuntti T, Ylikoski R, Hietanen M, Vataja R, Kaste M. Clinical determinants of poststroke dementia. Stroke 1998; 29: 75-81.

Powell AL, Cummings JL, Hill MA, Benson DF. Speech and language alterations in multi-infarct dementia. Neurology 1988; 38: 717-9.

Reichman W, Cummings J, McDaniel K, Flynn F, Gornbein J. Visuoconstructional impairment in dementia syndromes. Behav Neurol 1991; 4: 153-62. 
Reitan R, Wolfson D. The Halstead-Reitan Neuropsychological Test Battery. Tucson, AZ: Neuropsychology Press, 1985.

Ricker J, Axelrod B. Analysis of an oral paradigm for the Trail Making Test. Assessment 1994; 1: 47-51.

Ricker J, Axelrod B, Houtler B. Clinical validation of the oral Trail Making Test. Neuropsychiatry Neuropsychol Behav Neurol 1996; 9: 50-3.

Román GC, Tatemichi TK, Erkinjuntti T, et al. Vascular dementia: diagnostic criteria for research studies. Report of the NINDS-AIREN International Workshop. Neurology 1993; 43: 250-60.

Rosen WG, Mohs RC, Davis KL. A new rating scale for Alzheimer's disease. Am J Psychiatry $1984 ; 141: 1356-64$.

Ruff R. Ruff Figural Fluency Test. San Diego, CA: Neuropsychological Resources, 1988.

Spreen O, Strauss E. A compendium of neuropsychological tests: administration, norms, and commentary, 2nd ed. New York: Oxford, 1998.

Starkstein SE, Sabe L, Vazquez S, et al. Neuropsychological, psychiatric, and cerebral blood flow findings in vascular dementia and Alzheimer's disease. Stroke 1996; 27: 408-14.

Stern Y, Liu X, Albert M, et al. Modeling the influence of extrapyramidal signs on the progression of Alzheimer disease. Arch Neurol 1996a; 53: 1121-6.

Stern Y, Liu X, Albert M, et al. Application of a growth curve approach to modeling the progression of Alzheimer's disease. J Gerontol A Biol Sci Med Sci 1996b; 51: M179-84.

Tatemichi TK, Desmond DW, Mayeux R, et al. Dementia after stroke: baseline frequency, risks, and clinical features in a hospitalized cohort. Neurology 1992a; 42: 1185-93. 
Tatemichi TK, Desmond DW, Prohovnik I. Strategic infarcts in vascular dementia: a clinical and brain imaging experience. Drug Research 1995; 45: 371-85.

Tatemichi TK, Desmond DW, Prohovnik I, et al. Confusion and memory loss from capsular genu infarction: a thalamocortical disconnection syndrome?

Neurology 1992b; 42: 1966-79.

Tatemichi TK, Paik M, Bagiella E, Desmond DW, Pirro M, Hanzawa LK. Dementia after stroke is a predictor of long-term survival. Stroke 1994; 25: 1915-9.

Teng EL, Chui HC. The Modified Mini-Mental State (3MS) examination. J Clin Psychiatry 1987; 48: 314-8.

van Zagten M, Boiten J, Kessels F, Lodder J. Significant progression of white matter lesions and small deep (lacunar) infarcts in patients with stroke. Arch Neurol 1996; 53: 650-5.

Villardita C. Alzheimer's disease compared with cerebrovascular dementia: neuropsychological similarities and differences. Acta Neurol Scand 1993; 87: 299-308.

Wechsler D. The Wechsler Adult Intelligence Scale - Revised. New York: The Psychological Corporation, 1981.

Wetzel L, Boll T. Short Category Test, Booklet Format. Los Angeles: Western Psychological Services, 1987.

World Health Organization. The ICD-10 classification of mental and behavioural disorders: diagnostic criteria for research. Geneva, Switzerland: World Health Organization, 1993.

Yoshitake T, Kiyohara Y, Kato I, et al. Incidence and risk factors of vascular dementia and Alzheimer's disease in a defined elderly Japanese population: the Hisayama Study. Neurology 1995; 45: 1161-8. 
Zimmer NA, Hayden S, Deidan C, Loewenstein DA. Comparative performance of mildly impaired patients with Alzheimer's disease and multiple cerebral infarctions on tests of memory and functional capacity. Int Psychogeriatr 1994; 6: 143-54. 